it remains prudent to target lipid levels according to established guidelines. Of course, it also remains important to recommend increased exercise, healthy food choices, and portion control and to help manage weight in patients with prediabetes levels of glycemia or metabolic syndrome.

Studies to define the risks of statin-induced diabetes and its underlying mechanisms are clearly necessary. But until more data are available, clinicians should monitor glucose or glycated hemoglobin in patients with multiple risk factors for diabetes who take statins, but they should continue to prescribe statins when indicated as part of a multifactorial approach to managing cardiovascular risk.

Disclosure forms provided by the author are available with the full text of this article at NEJM.org.

From Harvard Medical School and the Section of Clinical, Behavioral, and Outcomes Research, Joslin Diabetes Center - both in Boston.

This article (10.1056/NEJMp1203020) was published on April 25, 2012, at NEJM.org.

1. Kearney PM, Blackwell L, Collins R, et al. Efficacy of cholesterol-lowering therapy in
18,686 people with diabetes in 14 randomised trials of statins: a meta-analysis. Lancet 2008;371:117-25.

2. Ridker PM, Danielson E, Fonseca FA, et al. Rosuvastatin to prevent vascular events in men and women with elevated C-reactive protein. N EnglJ Med 2008;359:2195-207.

3. Rajpathak SN, Kumbhani DJ, Crandall J, Barzilai N, Alderman M, Ridker PM. Statin therapy and risk of developing type 2 diabetes: a meta-analysis. Diabetes Care 2009;32: 1924-9.

4. Sattar N, Preiss D, Murray HM, et al. Statins and risk of incident diabetes: a collaborative meta-analysis of randomised statin trials. Lancet 2010;375:735-42.

5. Preiss D, Seshasai SR, Welsh P, et al. Risk of incident diabetes with intensive-dose compared with moderate-dose statin therapy: a meta-analysis. JAMA 2011;305:2556-64. Copyright (c) 2012 Massachusetts Medical Society.

\title{
Measles in the 21st Century
}

\author{
E. Kim Mulholland, M.D., Ulla Kou Griffiths, M.Sc., and Robin Biellik, Ph.D.
}

\begin{abstract}
arely 20 years ago, such a high proportion of childhood deaths globally was attributable to measles that the going estimate of more than 1 million measles-related deaths per year was almost certainly an underestimate. Pediatric wards in the developing world were filled with

An interactive
graphic showing
incidence and vaccination
rates is available
at NEJM.org
patients with measles and its complications, and measles continued to be a major cause of blindness globally. All this occurred despite the remarkable progress that had been achieved during the 1980 s in bringing routine immunizations, including a single dose of measles vaccine, to the poorest countries of the world, culminating in the achievement of the global Universal Childhood Immunization goals in 1990. In the United States, where measles had been effectively controlled since 1982, a minor resurgence of the disease occurred between 1989 and 1991, resulting in 123 deaths and more than 11,000 hospitalizations. Almost half of all cases
\end{abstract}

were in older children or adults. The subsequent introduction of a two-dose vaccination strategy led to the elimination of measles in the United States by 2000 , although imported cases continue to feed small outbreaks. In 2011, $90 \%$ of the 222 cases reported in the United States were associated with imported cases.

During the 1990 s, routine immunization stagnated in many parts of the developing world, especially Africa. The increasing emphasis on controlling poliovirus through focused campaigns may have been a factor in this stagnation, although it did enable some countries to undertake measles-vaccination campaigns that led to variable levels of control. By 2000, the countries of the African Region of the World Health Organization (WHO) were reporting more than 500,000 measles cases per year. ${ }^{1}$ Global measles-related deaths were estimated at more than 700,000 per year, but there was considerable disagreement over these modeled estimates, and many experts be- lieved they were too high. Between 2000 and 2008, measles control improved markedly in all regions (see the interactive graphic, available with the full text of this article at NEJM.org). Indigenous measles transmission was interrupted in the Americas by 2002, and the number of reported cases in Africa in 2008 was less than $10 \%$ of the 2000 level, despite improved reporting methods. ${ }^{2}$ Control was being achieved through the addition of a second dose of measles vaccine, either within the routine schedule for countries with wellfunctioning programs or in targeted campaigns.

After 2005, a new pattern began to emerge, with some richer countries failing to maintain sufficient vaccination coverage to control the disease. In Western Europe, Switzerland, Germany, France, and Britain reported continuing measles transmission and declining vaccination coverage, associated with sensationalized reports of adverse events, objection to immunization among certain groups, and a marked in- 
crease in the activities of antivaccination lobbying groups. During the same period, China and Japan brought their measles incidence down to very low levels.

Regardless of what the true mortality rate was in 2000 , there is no doubt that by 2008 global measles-related deaths had declined markedly, to an estimated $164,000 .^{3}$ Measles-control activities had been outstandingly successful. It seemed to be only a matter of time before the world could feasibly take on the task of measles eradication. Advocates pointed to the success in the Americas as evidence of the feasibility of eradication, though opponents highlighted the extreme infectiousness of the virus, the lack of understanding of measles epidemiology, the survival of the virus in remote areas, and the seemingly insurmountable technical and financial difficulties facing polio-eradication efforts.

By 2008, the WHO and partners were struggling with polio eradication, which had missed its 2000 global target. On scientific and public health grounds, the feasibility, desirability, and timing of measles eradication should not be dependent on the ongoing polio-eradication effort. In practice, however, the two efforts are inextricably linked. Because the same donors that fund polio-eradication programs will be called on to support measles eradication, the shifting of resources could jeopardize polioeradication efforts. Some argue that if polio eradication is really feasible, it should be completed before measles-eradication efforts are launched; yet by 2008, continuing polio transmission in India, Nigeria, Pakistan, and other countries where the virus was endemic was leading to growing

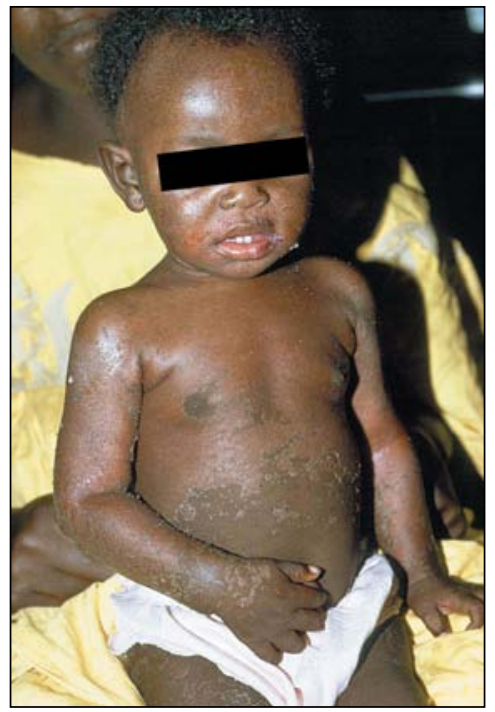

An African child with severe measles on day 10 of the disease.

skepticism about the feasibility of eradication.

Meanwhile, since 2008, the measles genie seems to have slipped out of the bottle in many regions. In Africa, measles control has deteriorated markedly, with the number of confirmed cases increasing from around 37,000 in 2008 to more than 172,000 in $2010 .{ }^{1}$ The distribution of cases follows the expected epidemiologic patterns: countries that had previously achieved good control report many cases in older children and adults, and countries with historically poor control, such as Nigeria, report that most cases occur in infancy and early childhood. A common feature in developing countries is the substantial proportion of cases occurring in the first year of life. The situation in Europe continues to be concerning as well, with measles incidence increasing in many industrialized countries. France alone reported over 15,000 measles cases, including 6 deaths, in 2011, according to the WHO.

In Africa, funding for measles control has fallen away since
2008, and the frequency and, in some cases, effectiveness of campaigns have declined. In Europe and parts of southern Africa, certain religious groups refuse immunization, and the result is concentrated pools of susceptible persons. In some African countries, outbreaks have occurred a short time after the implementation of campaigns, which suggests that the campaigns' quality has declined and that reported coverage may be exaggerated. To ensure that every child receives two doses of measles vaccine, both routine service delivery and campaigns must strive to achieve $100 \%$ coverage. Epidemiologic analysis is critical in order to determine the appropriate age groups to be covered in campaigns. In most countries, the incidence of disease is highest among children in the first year of life, whereas the proportion of cases occurring in children older than 5 years of age and in adults varies from country to country.

Infants under 9 months of age are traditionally regarded as too young to be vaccinated. Increasingly, however, babies are being born to mothers with minimal measles immunity, often owing to minimal natural boosting after childhood immunization. Such mothers pass less-protective immunity to their infants, who are therefore protected for a shorter period, and it has been proposed that these infants be vaccinated at a younger age. ${ }^{4} \mathrm{~A}$ recent trial of measles immunization at 4 months of age in Guinea-Bissau showed adequate protective efficacy. ${ }^{5}$ If these results can be replicated elsewhere, it would be appropriate to revisit the recommended age of first measles vaccination in order to close the current window of susceptibility. 
Although global measles control seems to be struggling, the polio situation is looking somewhat more promising. On January 13,2012 , India reported that it had been 12 months since the last wild-virus poliomyelitis case was confirmed in that country. However, 2011 saw increased numbers of new polio cases in Pakistan (197), Chad (131), Nigeria (57), and Afghanistan (80), according to the Global Polio Eradication Initiative. Polio eradication remains an elusive target, although there is growing optimism that it may eventually be achieved. If and when that occurs, the overall financial costs plus the opportunity costs will have exceeded the initial estimates many times over - a point that is not likely to be lost on funding agencies. These figures will be essential for calculating realistic costs of measles eradication, which should be analyzed and weighed against the substantial future health and economic benefits such an initiative could bring. In the shorter term, however, until greater measles control is achieved, particularly in Western Europe and Africa, health professionals in the United States can anticipate more small outbreaks among susceptible groups due to imported measles virus.

Disclosure forms provided by the authors are available with the full text of this article at NEJM.org.
From the London School of Hygiene and Tropical Medicine, London (E.K.M., U.K.G.); Menzies School of Health Research, Darwin, NT, and Murdoch Childrens Research Institute, Parkville, VIC (E.K.M.) - both in Australia; and Geneva (R.B.).

1. Measles outbreaks and progress towards meeting measles pre-elimination goals: WHO African Region, 2009-2010. Wkly Epidemiol Rec 2011;86:129-40.

2. Wolfson LJ, Strebel PM, Gacic-Dobo M, Hoekstra EJ, McFarland JW, Hersh BS. Has the 2005 measles mortality reduction goal been achieved? A natural history modelling study. Lancet 2007;369:191-200.

3. Moss WJ, Griffin DE. Measles. Lancet 2012; 379:153-64.

4. Mulholland K. Measles and pertussis in developing countries with good vaccine coverage. Lancet 1995;345:305-7.

5. Martins CL, Garly M-L, Balé C, et al. Protective efficacy of standard EdmonstonZagreb measles vaccination in infants aged 4.5 months: interim analysis of a randomised clinical trial. BMJ 2008;337:a661.

Copyright (c) 2012 Massachusetts Medical Society. 